\title{
The Influence of Sitagliptin on Treatment-Related Quality of Life in Patients with Type 2 Diabetes Mellitus Receiving Insulin Treatment: A Prespecified Sub-Analysis
}

\author{
Tomoya Mita (D) - Naoto Katakami - Toshihiko Shiraiwa - Hidenori Yoshii • \\ Masahiko Gosho · Hitoshi Ishii · Iichiro Shimomura · Hirotaka Watada • \\ SPIKE Trial Site Investigators
}

Received: March 22, 2017 / Published online: May 17, 2017

(C) The Author(s) 2017. This article is an open access publication

\section{ABSTRACT}

Introduction: Treatment-related quality of life (QOL) is an important aspect of diabetes management. Here, we investigated the influence of sitagliptin, a dipeptidyl peptidase- 4 inhibitor, on treatment-related QOL in patients with type 2 diabetes mellitus treated with insulin.

Methods: This was a prespecified sub-analysis of the Sitagliptin Preventive Study of

Enhanced content To view enhanced content for this article go to http://www.medengine.com/Redeem/ 7848F060253101E5.

SPIKE Trial Site Investigators involved in this study are listed in the "Acknowledgements".

T. Mita $(\bowtie) \cdot$ H. Watada

Department of Metabolism and Endocrinology, Juntendo University Graduate School of Medicine, Hongo 2-1-1, Bunkyo-ku, Tokyo 113-8421, Japan e-mail: tom-m@juntendo.ac.jp

N. Katakami · I. Shimomura

Department of Metabolic Medicine, Osaka

University Graduate School of Medicine, 2-2,

Yamadaoka, Suita, Osaka 565-0871, Japan

N. Katakami

Department of Metabolism and Atherosclerosis,

Osaka University Graduate School of Medicine, 2-2,

Yamadaoka, Suita, Osaka 565-0871, Japan

T. Shiraiwa

Shiraiwa Medical Clinic, 4-10-24 Houzenji,

Kashiwara, Osaka 582-0005, Japan
Intima-Media Thickness Evaluation (SPIKE). The study population consisted of 71 subjects in the sitagliptin group, and 62 subjects in the conventional group who were treated with insulin. Patients of the sitagliptin group were started on sitagliptin in addition to ongoing insulin therapy. In the conventional group, either increasing the dose of current insulin therapy or the addition of oral hypoglycemic agents other than dipeptidyl peptidase- 4 inhibitors was allowed to achieve glycemic control. Treatment-related QOL was evaluated before and 104 weeks after the initiation of the study using the Diabetes Therapy-Related QOL Questionnaire 7 (DTR-QOL7).

Results: Forty-five out of 71 subjects in the sitagliptin group and 41 out of 62 subjects in

\section{H. Yoshii}

Department of Medicine, Diabetology and Endocrinology, Juntendo Tokyo Koto Geriatric Medical Center, Shinsuna 3-3-20, Koto-ku, Tokyo 136-0075, Japan

\section{Gosho}

Department of Clinical Trial and Clinical Epidemiology, Faculty of Medicine, University of Tsukuba, 1-1-1, Tennodai, Tsukuba, Ibaraki 305-8575, Japan

H. Ishii

Department of Diabetology, Nara Medical University, 840 Shijo, Kashihara, Nara 634-8552, Japan 
the conventional group filled out the QOL questionnaire at week 104. The DTR-QOL7 score at week 104 was significantly increased from baseline in the sitagliptin group, while that in the conventional group was not changed. However, the changes in score did not differ between the two groups. Change in HbA1c was negatively associated with change in score.

Conclusions: Our data suggest that sitagliptin added to insulin treatment was comparable to other treatments in terms of its impact on treatment-related QOL.

Clinical Trial Registration: ClinicalTrials.gov identifier: UMIN000007396.

Funding: Mitsubishi Tanabe Pharma Co., Ono Pharmaceutical Co., and Novo Nordisk.

Keywords: Sitagliptin; $\quad$ Treatment-related quality of life; Type 2 diabetes mellitus

\section{INTRODUCTION}

One of the main goals in the management of type 2 diabetes (T2DM) is to maintain quality of life (QOL). Effective treatment needs to achieve these goals by taking into account many factors, including age, disease duration, glycemic control status, physical status, and diabetic complications. In terms of choosing oral hypoglycemic agents, consideration of treatment-related QOL is important because it has been recognized as an important factor associated with patient motivation and adherence [1]. This is crucial since poor adherence to T2DM treatment has been shown to be associated with poor glycemic control and increased risk of mortality [2]. The American Diabetes Association therefore emphasized the importance of considering patient preference in addition to efficacy, hypoglycemic risk, impact on weight, potential side effects, and cost [3].

Hypoglycemia and weight gain are common side effects of treatment for T2DM [4] and the major barrier to achieving optimal glycemic control, especially with insulin therapy. In this regard, treatment with insulin plus metformin [5] and $\alpha$-glucosidase inhibitors [6], but not pioglitazone [7] or sulfonylurea [8], were advantageous in avoiding both weight gain and hypoglycemia. However, there are treatment-limiting side effects with $\alpha$-glucosidase inhibitors or metformin such as gastrointestinal symptoms $[6,9]$. These treatment-limiting side effects may be associated with reduction in treatment-related QOL in patients. Dipeptidyl peptidase-4 (DPP-4) inhibitors reduced blood glucose levels through increasing insulin secretion and suppressing glucagon release and are generally safe and well tolerated without increasing body weight $[10,11]$. As a result of these characteristics, DPP-4 inhibitors are widely prescribed in Japan. In addition, sitagliptin was shown to improve QOL in patients treated with oral hypoglycemic agents in a single-arm study [12].

In addition, DPP-4 inhibitors are sometimes administered to patients receiving insulin; however, treatment-related QOL in this population has not yet been elucidated. Recently, we conducted the Sitagliptin Preventive Study of Intima-Media Thickness Evaluation (SPIKE) study to investigate the effect of sitagliptin, a DPP-4 inhibitor, on carotid atherosclerosis and reported that sitagliptin slowed the progression of carotid intima-media thickness in insulin-treated T2DM patients [13]. However, there are few studies that investigate the effect of DPP-4 inhibitors on QOL in patients treated with insulin while recent studies demonstrated that the addition of DPP-4 inhibitors to insulin therapy improved blood glucose levels without increased risk for hypoglycemia and increasing body weight $[13,14]$. The present study was originally planned as a sub-analysis of the SPIKE study to investigate the effect of sitagliptin on treatment-related QOL in patients treated with insulin.

\section{METHODS}

\section{Study Population}

We performed a sub-analysis of the SPIKE study, whose methods were described in detail previously [15]. Briefly, a total of 282 insulin-treated Japanese T2DM patients free of a past history of apparent cardiovascular disease were randomly 
allocated to either the sitagliptin group ( $n=142)$ or the conventional treatment group (using oral hypoglycemic agents other than the DPP-4 inhibitor $(n=140)$. Patients were included regardless of the number of insulin injections or type of insulin. Randomization is performed using a dynamic allocation method based on the number of insulin injections, with/without pioglitazone, age, and gender. In the conventional treatment group, either increasing the dose of current therapy (e.g., insulin) or the addition of sulfonylurea, glinide, and $\alpha$-glucosidase inhibitors is allowed with the goal of achieving the target value specified in the Treatment Guide for Diabetes (usually HbA1c level less than $6.9 \%$ and/or fasting blood glucose less than $130 \mathrm{mg} / \mathrm{dl}$ and/or $2 \mathrm{~h}$ postprandial blood glucose less than $180 \mathrm{mg} / \mathrm{dl}$ ) [16] at each medical provider's discretion. The addition of other DPP-4 inhibitors and glucagon-like peptide- 1 analogues is banned in the control group. The dose adjustment and addition of metformin and pioglitazone are banned in both groups during the study. In case of hypoglycemia, the dose of insulin and/or oral hypoglycemic agents is titrated. All patients who agreed to participate were entered into the study and signed written informed consent. The SPIKE study was registered with the University Hospital Medical Information Network Clinical Trials Registry (UMIN000007396) and meets the requirements of the International Committee of Medical Journal Editors.

\section{Compliance with Ethics Guidelines}

All procedures followed were in accordance with the ethical standards of the responsible committee on human experimentation (institutional and national) and with the Helsinki Declaration of 1964, as revised in 2013. Informed consent was obtained from all patients for being included in the study.

\section{Diabetes Therapy-Related QOL Questionnaire (DTR-QOL) 7}

The DTR-QOL is a reliable and valid questionnaire developed by Ishii; it is a 29-item, self-administered assessment with four primary factors [17]. As a result of practical constraints we created a short version, the DTR-QOL7. This involved selecting six questions from the original 29 items by considering relationships among items based on the results of the original study. Of the 23 excluded items, we chose to include one $(\mathrm{Q} 2)$ that asked about weight gain with treatment because this factor is likely to have a major impact on QOL based on clinical experience. Unfortunately, the way of selecting seven questions from the original 29 items was not based on technical or statistical rationales. However, we confirmed that all six items other than Q2 seemed to be included in the same domain, suggesting that the structure of the DTR-QOL7 was relatively consistent in the original study. The total scores of DTR-QOL7 except Q2 had high internal consistency based on Cronbach's alpha coefficients (data not shown) and were highly associated with the total scores of the original 29 items. The items included are shown in Table 1 . The response to each question consisted of a 7-point Likert-type scale that ranged from 1 (strongly agree) to 7 (strongly disagree). The scales of Q5, Q6, and Q7 were reversed so that 7 represented the highest QOL score. The total score, after simple addition of each item score, was converted to a range from 0 to 100 (best-case response $=100$; worst-case response $=0$ ). The score of Q2 was evaluated separately. We treated missing values according to the original DTR-QOL [17]. We measured the DTR-QOL7 at baseline and at 104 weeks.

\section{Statistical Analysis}

Results are presented as mean $\pm \mathrm{SD}$, median (quantile 1 and quantile 3), or number (proportion) of patients. Factor analysis was performed on the seven items to investigate whether the structure of the DTR-QOL7 was consistent. Variables with factor loading of at least 0.30 were considered for interpretation. The internal consistency of the total score and each domain of the DTR-QOL7 was assessed using Cronbach's alpha coefficient. An alpha of at least 0.70 is considered acceptable for the 
Table 1 DTR-QOL7 questionnaire

Q1. I am constantly concerned about time to manage my current diabetes treatment

Q2. I am bothered by weight gain with my current diabetes treatment

Q3. I am sometimes bothered by low blood glucose

Q4. I am worried about high blood glucose

Q5. Overall, I am satisfied with my current blood sugar control

Q6. With my current diabetes treatment, I am confident that I can maintain good blood glucose control

Q7. With regards to diabetes treatment, I am satisfied with current treatment methods

Table 2 Clinical characteristics of patients in the sitagliptin and conventional treatment groups

\begin{tabular}{|c|c|c|c|}
\hline Parameters & $\begin{array}{l}\text { Sitagliptin treatment } \\
\text { group }(n=71)\end{array}$ & $\begin{array}{l}\text { Conventional treatment } \\
\text { group }(n=62)\end{array}$ & $P$ value \\
\hline Age (years) & $62.6 \pm 11.7$ & $63.6 \pm 9.5$ & 0.59 \\
\hline Gender (male) (\%) & $40(56.3)$ & $31(50)$ & 0.49 \\
\hline Duration of diabetes (years) & $16.8 \pm 8.3$ & $17.2 \pm 8.3$ & 0.77 \\
\hline eGFR $\left(\mathrm{mL} / \mathrm{min} / 1.73 \mathrm{~m}^{2}\right)$ & $77.8 \pm 20.8$ & $79.5 \pm 22.3$ & 0.65 \\
\hline Body mass index $\left(\mathrm{kg} / \mathrm{m}^{2}\right)$ & $25.7 \pm 4.4$ & $25.3 \pm 4.0$ & 0.57 \\
\hline HbAlc (\%) & $8.3 \pm 1.2$ & $8.0 \pm 1.1$ & 0.15 \\
\hline Total daily insulin dosage (IU/day) & $32.3 \pm 22.8$ & $29.4 \pm 22.3$ & 0.46 \\
\hline Time of insulin injections (times/day) & $3.0 \pm 1.1$ & $3.1 \pm 1.0$ & 0.77 \\
\hline \multicolumn{4}{|l|}{ Type of insulin } \\
\hline Prandial insulin (yes) & $41(57.7)$ & $39(62.9)$ & 0.60 \\
\hline Premixed insulin (yes) & $35(49.3)$ & $18(29)$ & 0.02 \\
\hline Basal insulin (yes) & $31(43.7)$ & $33(53.2)$ & 0.30 \\
\hline \multicolumn{4}{|l|}{ Use of oral glucose-lowering agents } \\
\hline Metformin (yes) & $31(43.7)$ & $25(35.5)$ & 0.38 \\
\hline Sulfonylurea (yes) & $11(15.5)$ & $8(12.9)$ & 0.81 \\
\hline Glinides (yes) & $0(0)$ & $0(0)$ & - \\
\hline Thiazolidinediones (yes) & $9(12.7)$ & $4(6.5)$ & 0.26 \\
\hline$\alpha$-Glucosidase inhibitor (yes) & $31(43)$ & $24(38.7)$ & 0.60 \\
\hline
\end{tabular}

Data are number (\%) of patients or mean $\pm \mathrm{SD}$

HbAlc glycated hemoglobin A1c, eGFR estimated glomerular filtration rate

purpose of group comparisons [18]. Baseline and follow-up group comparisons were assessed with the Student's $t$ test or Wilcoxon's rank sum test for continuous variables and Fisher's exact test for categorical variables. Changes from baseline to treatment visits were assessed with one-sample $t$ test or Wilcoxon's signed rank test for continuous variables and McNemar's test for 
binary variables within the group. Differences in delta change in score of QOL from baseline to 104 weeks between groups were analyzed with analysis of covariance adjusted for score at baseline. All statistical tests were two-sided with a 5\% significance level. All analyses were performed using the SAS software version 9.4 (SAS Institute, Cary, NC).

\section{RESULTS}

\section{Baseline Characteristics}

Among patients in the original study, 71 in the sitagliptin group and 62 in the conventional group completed the DTR-QOL questionnaire at baseline. Baseline clinical characteristics were comparable between the two groups (Table 2). At week 104, 45 in the sitagliptin group and 41 in the conventional group completed the DTR-QOL questionnaire. Consequently, 47 subjects did not complete the DTR-QOL questionnaire. In the comparison of clinical characteristics at baseline between subjects who completed the DTR-QOL questionnaire and those who did not, there were statistical significant differences in total daily insulin dosage, the use of prandial insulin, and the use of metformin and $\alpha$-glucosidase inhibitors between subjects who completed the DTR-QOL questionnaire and those who did not (Table 3). On the other hand, there were no significant differences in clinical parameters at baseline between patients who completed the

Table 3 Baseline clinical characteristics of patients who completed questionnaire and who did not complete questionnaire

\begin{tabular}{|c|c|c|c|}
\hline Parameters & $\begin{array}{l}\text { Completion of questionnaire } \\
\text { at week } 104(n=86)\end{array}$ & $\begin{array}{l}\text { No completion of questionnaire } \\
\text { at week } 104(n=47)\end{array}$ & $P$ value \\
\hline Age (years) & $63.9 \pm 10.0$ & $61.5 \pm 11.8$ & 0.22 \\
\hline Gender (male) (\%) & $44(51.2)$ & $27(57.4)$ & 0.59 \\
\hline Body mass index $\left(\mathrm{kg} / \mathrm{m}^{2}\right)$ & $25.7 \pm 4.1$ & $25.1 \pm 4.5$ & 0.37 \\
\hline Duration of diabetes (years) & $17.7 \pm 8.6$ & $15.6 \pm 7.7$ & 0.16 \\
\hline HbAlc (\%) & $8.2 \pm 1.2$ & $8.1 \pm 1.2$ & 0.50 \\
\hline $\mathrm{eGFR}\left(\mathrm{mL} / \mathrm{min} / 1.73 \mathrm{~m}^{2}\right)$ & $77.4 \pm 20.6$ & $80.7 \pm 22.9$ & 0.39 \\
\hline Total daily insulin dosage (IU/day) & $33.0 \pm 24.1$ & $27.3 \pm 18.8$ & 0.046 \\
\hline Time of insulin injections (times/day) & $3.2 \pm 1.0$ & $2.8 \pm 1.1$ & 0.16 \\
\hline \multicolumn{4}{|l|}{ Type of insulin } \\
\hline Prandial insulin (yes) & $58(67.4)$ & $22(46.8)$ & 0.026 \\
\hline Premixed insulin (yes) & $35(49.3)$ & $21(44.7)$ & 0.46 \\
\hline Basal insulin (yes) & $42(48.8)$ & $22(46.8)$ & 0.86 \\
\hline \multicolumn{4}{|l|}{ Use of oral glucose-lowering agents } \\
\hline Metformin (yes) & $25(25.9)$ & $28(59.6)$ & $<0.001$ \\
\hline Sulfonylurea (yes) & $14(16.3)$ & $5(10.6)$ & 0.45 \\
\hline Glinides (yes) & $0(0)$ & $0(0)$ & - \\
\hline Thiazolidinediones (yes) & $10(11.6)$ & $3(6.4)$ & 0.38 \\
\hline$\alpha$-Glucosidase inhibitors (yes) & $21(24.4)$ & $34(72.3)$ & $<0.001$ \\
\hline
\end{tabular}

Data are number (\%) of patients or mean $\pm S D$

HbAlc glycated hemoglobin A1c, eGFR estimated glomerular filtration rate 
Table 4 Comparison of clinical parameters at baseline and week 104

\begin{tabular}{|c|c|c|c|c|c|c|}
\hline \multirow[t]{2}{*}{ Parameters } & \multicolumn{2}{|c|}{$\begin{array}{l}\text { Sitagliptin treatment } \\
\text { group }(n=45)\end{array}$} & \multicolumn{2}{|c|}{$\begin{array}{l}\text { Conventional treatment } \\
\text { group }(n=41)\end{array}$} & \multicolumn{2}{|c|}{$P$ value (intergroup) } \\
\hline & Baseline & Week 104 & Baseline & Week 104 & Baseline & Week 104 \\
\hline Age (years) & $62.6 \pm 11.9$ & - & $65.4 \pm 7.2$ & - & 0.19 & - \\
\hline Gender (male) (\%) & $25(55.6)$ & - & $19(46.3)$ & - & 0.52 & - \\
\hline Body mass index $\left(\mathrm{kg} / \mathrm{m}^{2}\right)$ & $26.1 \pm 4.4$ & $25.8 \pm 4.5$ & $25.3 \pm 3.7$ & $25.6 \pm 4.7$ & 0.37 & 0.82 \\
\hline Duration of diabetes (years) & $17.1 \pm 8.7$ & - & $18.4 \pm 8.4$ & - & 0.50 & \\
\hline HbAlc (\%) & $8.4 \pm 1.2$ & $7.7 \pm 1.7^{* *}$ & $8.0 \pm 1.1$ & $7.7 \pm 1.3$ & 0.21 & 0.51 \\
\hline $\mathrm{eGFR}\left(\mathrm{mL} / \mathrm{min} / 1.73 \mathrm{~m}^{2}\right)$ & $78.3 \pm 21.3$ & $73.6 \pm 25.8^{* *}$ & $76.4 \pm 20.0$ & $73.5 \pm 21.2$ & 0.67 & 0.98 \\
\hline Total daily insulin dosage (IU/day) & $3.2 \pm 1.1$ & $2.9 \pm 1.3$ & $3.2 \pm 0.9$ & $3.2 \pm 1.0$ & 0.77 & 0.35 \\
\hline Time of insulin injections (times/day) & $34.5 \pm 24.6$ & $30.2 \pm 22.7^{*}$ & $31.4 \pm 23.8$ & $29.0 \pm 19.7$ & 0.56 & 0.79 \\
\hline \multicolumn{7}{|l|}{ Type of insulin } \\
\hline Prandial insulin (yes) & $30(66.7)$ & $28(65.1)$ & $28(68.7)$ & $28(68.7)$ & 1.00 & 0.82 \\
\hline Premixed insulin (yes) & $18(40.0)$ & $15(34.9)$ & $14(34.1)$ & $11(26.8)$ & 0.66 & 0.48 \\
\hline Basal insulin (yes) & $24(53.3)$ & $25(58.1)$ & $18(43.9)$ & $23(56.1)$ & 0.40 & 1.00 \\
\hline \multicolumn{7}{|l|}{ Use of oral glucose-lowering agents } \\
\hline Metformin (yes) & $15(33.3)$ & $15(33.3)$ & $10(24.4)$ & $9(22.0)$ & 0.48 & 0.34 \\
\hline Sulfonylurea (yes) & $8(17.8)$ & $8(17.8)$ & $6(14.6)$ & $7(17.1)$ & 0.79 & 1.00 \\
\hline Glinides (yes) & $0(0)$ & $1(2.2)$ & $0(0)$ & $1(2.4)$ & - & 1.00 \\
\hline Thiazolidinediones (yes) & $8(17.8)$ & $8(17.8)$ & $2(4.9)$ & $2(4.9)$ & 0.09 & 0.09 \\
\hline$\alpha$-Glucosidase inhibitor (yes) & $12(26.7)$ & $12(24.4)$ & $9(22)$ & $9(22)$ & 0.63 & 0.80 \\
\hline
\end{tabular}

Data are number (\%) of patients or mean \pm SD values

Changes from baseline to week 104 were assessed with by one-sample $t$ test or Wilcoxon's signed rank test for continuous variables and McNemar's test for binary variables within the group: ${ }^{*} P<0.05,{ }^{* *} P<0.01$

$H b A 1 c$ glycated hemoglobin A1c, $e G F R$ estimated glomerular filtration rate

DTR-QOL questionnaire in the sitagliptin group and those who did in the conventional group (Table 4). The addition of sitagliptin to insulin therapy significantly reduced HbA1c level from baseline to week 104 (Table 4). The change in HbA1c level from baseline to week 104 was numerically greater in subjects who completed the DTR-QOL questionnaire of the sitagliptin group than in subjects who completed it in the conventional group; however, there was no significant difference between the two groups $(-0.6 \pm 1.4$ vs. $-0.3 \pm 1.0 \%, P=0.23)$. On the other hand, there were no differences between the two groups in change in body mass index $(-0.3 \pm 2.1$ vs. $0.3 \pm 2.5 \mathrm{~kg})$ or in the average number of hypoglycemic episodes [0.0 $(0.0,0.4)$ vs. $0.0(0.0,0.3)$ times/month/person] over 104 weeks (data not shown).

\section{Factor Analysis and Internal Consistency}

Factor analysis with promax rotation was performed to investigate the structure of the DTR-QOL7 (Table 5). As expected, all six items other than Q2 seemed to be included in the 
Table 5 Seven items on the DTR-QOL7 and factor analysis with promax rotation $(n=131)$

\begin{tabular}{lc}
\hline Question number & Factor 1 \\
\hline Q1 & $\mathbf{0 . 3 6}$ \\
Q2 & -0.02 \\
Q3 & $\mathbf{0 . 4 3}$ \\
Q4 & $\mathbf{0 . 4 6}$ \\
Q5 & $\mathbf{0 . 6 8}$ \\
Q6 & $\mathbf{0 . 7 6}$ \\
Q7 & $\mathbf{0 . 5 8}$ \\
\hline
\end{tabular}

Individual question items with a factor loading of $>|0.3|$ are shown in bold

same domain, suggesting that the structure of the DTR-QOL7 was relatively consistent. According to internal consistency analysis of the DTR-QOL7, all six items except for Q2 showed moderate internal consistency, with a Cronbach's alpha coefficient of 0.71 . The result of the factor analysis at 104 weeks was almost similar to that at baseline (data not shown).

\section{Temporal Change in DTR-QOL7 Scores}

Both at baseline and at 104 weeks, there were no differences between the two groups in the total DTR-QOL7 score (excluding Q2) or in the score of each individual question (Q1-Q7), as shown in Table 6. The total DTR-QOL7 score and the scores of Q5 and Q6 were significantly increased at 104 weeks compared to baseline in the sitagliptin group. In the conventional group there were no significant score changes over time. There were no significant differences between the two groups in changes from baseline to week 104 of the total DTR-QOL7 score or the score of any individual question (Q1-Q7).

We investigated the relationship between changes in total DTR-QOL7 score and changes in various parameters. The change in HbA1c at week 104 was negatively associated with change in total DTR-QOL7 score (Spearman's correlation coefficient, $r=-0.35, P<0.001)$; age at baseline, gender, body mass index at baseline, duration of T2DM, and type of treatment showed no association (data not shown). Also, the occurrence of hypoglycemia showed negative, non-significant association with change in total DTR-QOL7 score $(r=-0.21, P=0.05)$.

\section{DISCUSSION}

In this study, sitagliptin treatment increased the score of DTR-QOL7 from baseline while conventional treatment resulted in no change. On the other hand, there were no significant differences in the use of oral glucose-lowering agents other than DPP-4 inhibitors between two groups. Thus, increase in the score of DTR-QOL7 may be associated with sitagliptin used as an add-on therapy to insulin itself. However, the change in DTR-QOL7 score did not differ between the two groups. Taken together, these results may suggest that sitagliptin added to insulin treatment was comparable to other treatments in terms of its impact on treatment-related QOL.

A previous report demonstrated that glycemic control was associated with higher treatment-related QOL [17]. In this study, change in HbA1c level was negatively associated with change in the total DTR-QOL7 score. Thus, it is reasonable to hypothesize that sitagliptin treatment improved QOL by increasing patient satisfaction with aspects of treatment related to glycemic control (Q5 and Q6). On the other hand, adverse effects and patient acceptance of treatment may worsen treatment-related QOL. In particular, more frequent hypoglycemic episodes have a harmful effect on QOL $[19,20]$. In addition, weight gain caused by diabetes treatment may be an undesired feature and negatively affects QOL. With respect to these issues, sitagliptin treatment did not worsen QOL as shown by constant scores on Q2 and Q3. These findings are reasonable considering that sitagliptin treatment did not increase body weight or risk of hypoglycemia compared to conventional treatment.

The present study has certain limitations. First, the study was a sub-analysis that included a relatively small sample from the original study because the questionnaire was completed on a voluntary basis. This may have caused selection 


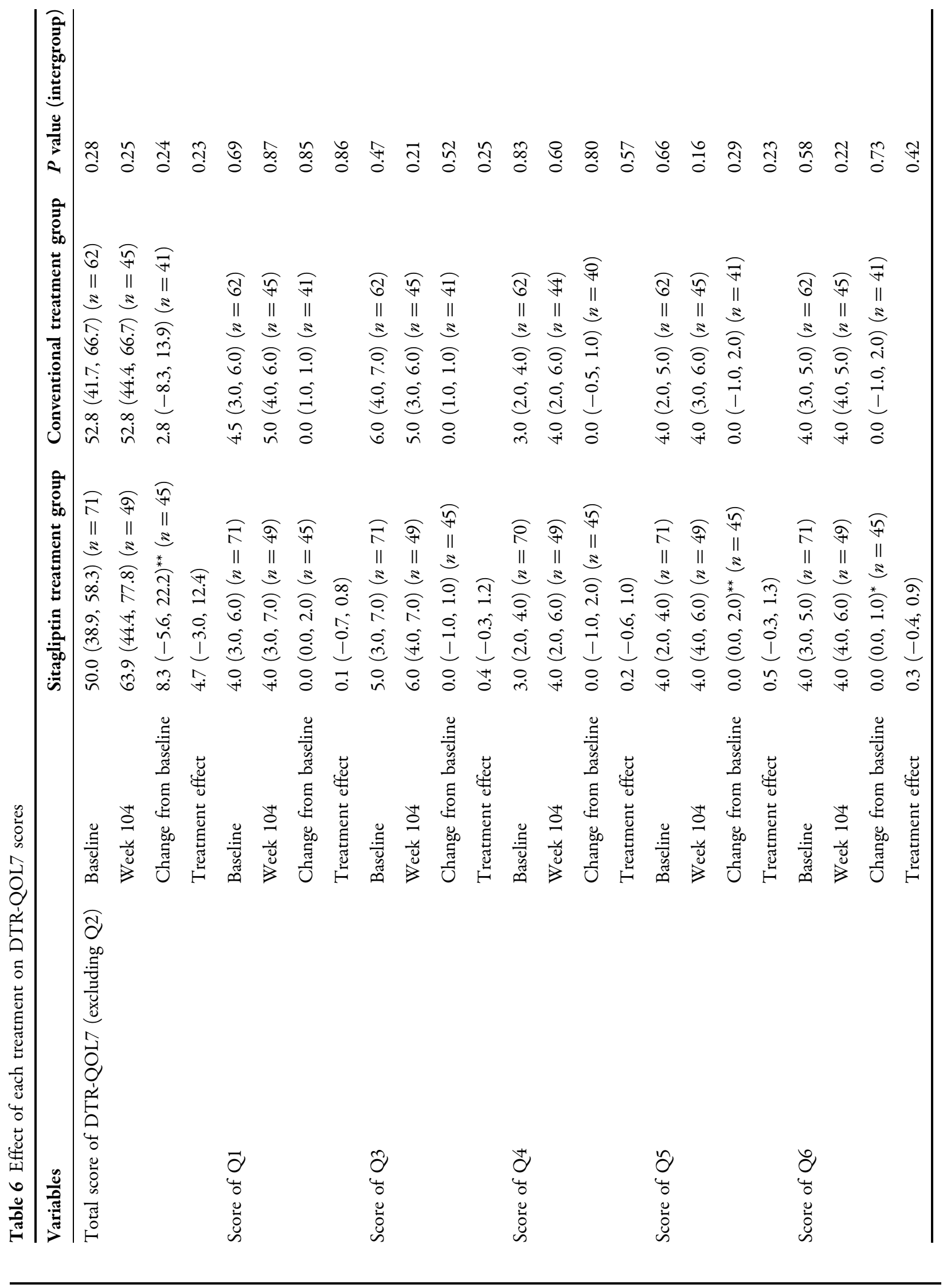




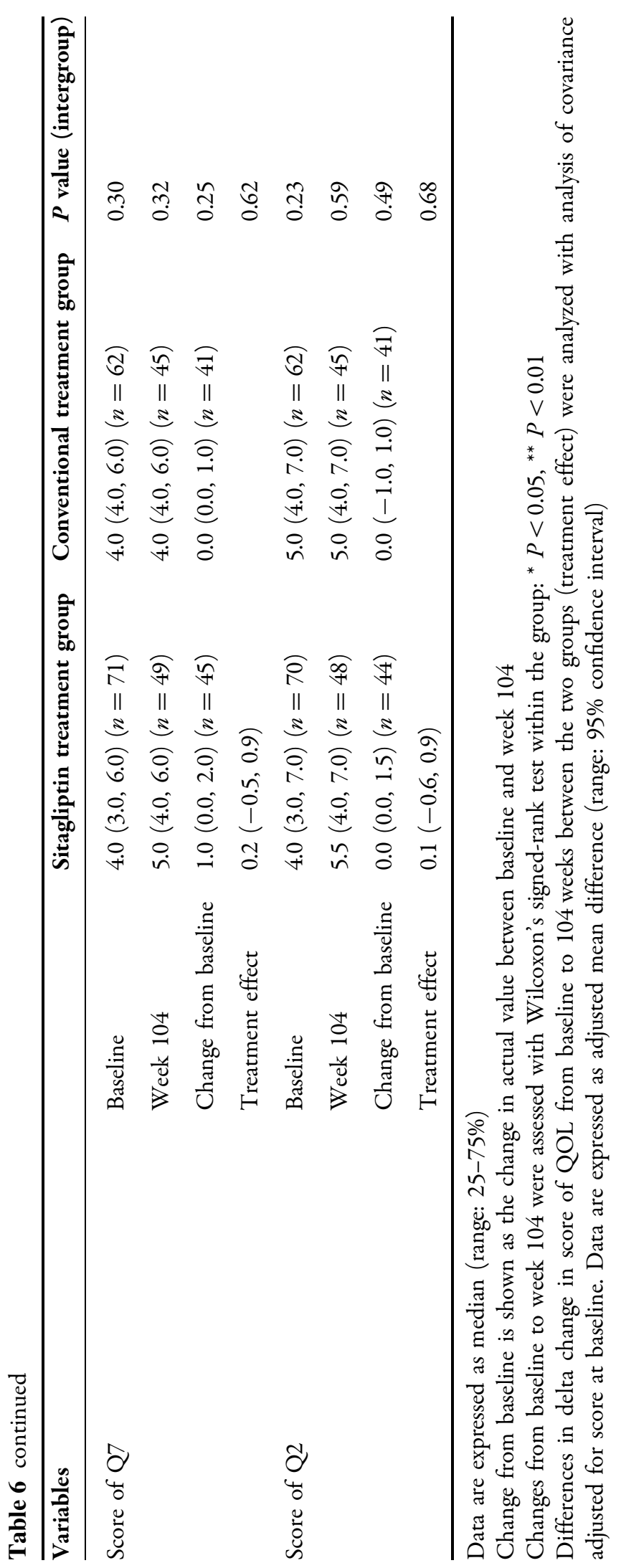


bias. In addition, there were statistically significant differences in total daily insulin dosage, the use of prandial insulin, and the use of metformin and $\alpha$-glucosidase inhibitors between subjects who completed the DTR-QOL questionnaire and those who did not. Although we could not rule out the possibility that obtained data did not reflect the characteristics of the original population, there were no significant differences in clinical parameters at baseline between subjects who completed the DTR-QOL questionnaire in the two groups. Second, we evaluated treatment-related QOL using only the DTR-QOL7. Its small number of questions is a weakness in terms of evaluating the many ways that diabetes treatment can influence QOL. However, we confirmed that the structure of the DTR-QOL7 was consistent and that it had moderate internal consistency. Further studies are required to confirm our findings.

\section{CONCLUSIONS}

Our data suggest that sitagliptin treatment did not worsen treatment-related QOL compared to conventional treatment, at least when used as an add-on therapy to insulin.

\section{ACKNOWLEDGEMENTS}

Financial support was provided by the Japan Society for Patient-Reported Outcome research fund from Mitsubishi Tanabe Pharma Co., Ono Pharmaceutical Co., and Novo Nordisk. The funder had no role in study design, data collection and analysis, decision to publish, or preparation of the manuscript. They did not fund the journal's article processing charges or medical writing assistance. All named authors meet the International Committee of Medical Journal Editors (ICJME) criteria for authorship for this manuscript. All authors had full access to all of the data in this study and take complete responsibility for the integrity of the data and accuracy of the data analysis, and have given final approval for the version to be published. We confirm that all authors had access to the study data that support this publication. The authors thank all the clinical staff for their assistance with the execution and completion of the clinical trial.

SPIKE Trial Site Investigators. The SPIKE study is a multicenter collaboration. In addition to the listed authors, the following SPIKE Study Investigators were involved in this study: Jiyugaoka Medical Clinic: Hiroki Yokoyama; Juntendo Tokyo Koto Geriatric Medical Center (Department of Medicine, Diabetology \& Endocrinology): Kanae Ishida, Noriko Inagaki, Tomio Onuma, Keiko Yamashiro, Junko Yokota; Juntendo University Graduate School of Medicine (Department of Metabolism \& Endocrinology): Fuki Ikeda, Koji Komiya, Yuko Sakurai, Motoyuki Tamaki; Kansai Rosai Hospital: Daisuke Azuma, Isao Hayashi, Isao Hayashi, Tsunehiko Yamamoto; Misaki Naika Clinic: Nobuichi Kuribayashi; Naka Memorial Clinic: Hidenori Isida, Takeshi Osonoi, Miyoko Saito; Osaka General Medical Center: Masahiro Hatazaki, Ryutaro Kataoka, Yutaka Umayahara; Osaka Police Hospital: Keisuke Kosugi, Ken'ya Sakamoto, Kazutomi Yoshiuchi; Osaka University Graduate School of Medicine (Department of Metabolic Medicine): Hideaki Kaneto, Taka-aki Matsuoka, Ken Ohya, Sae Uno; Sasebo Chuo Hospital: Kazunari Matsumoto, Fumi Mori, Yoshitaka Mori; Shiraiwa Medical Clinic: Toshihiko Shiraiwa.

Disclosures. Tomoya Mita received research funds from MSD and Takeda Pharma K.K. and has received lecture fees from AstraZeneca K.K., Boehringer Ingelheim, Eli Lilly, Kowa Pharmaceutical Co., Mitsubishi Tanabe Pharma Co., MSD, Ono Pharmaceutical Co., and Takeda Pharmaceutical Co. Naoto Katakami holds an endowed chair (Department of Metabolism and Atherosclerosis) established by funds from Kowa Pharmaceutical Co., has received research funds from MSD and lecture fees from Arkray Co. Ltd., Astellas Pharma Inc., Boehringer Ingelheim, Daiichi Sankyo Inc., Dainippon Sumitomo Pharma Co., Eli Lilly, Kowa Pharmaceutical Co., Kyowa Hakko Kirin Co. Ltd., Mitsubishi Tanabe Pharma Co., Novo Nordisk Pharma, Ono Pharmaceutical Co., Takeda Pharmaceutical Co., Sanofi-Aventis, and Shionogi \& Co. Toshihiko Shiraiwa has received lecture fees from 
Boehringer Ingelheim, Sanofi-Aventis, Novo Nordisk Pharma, Novartis Pharmaceuticals, Eli Lilly, Abbott Japan, Takeda Pharmaceutical Co., Sanwakagaku Kenkyusho, Mitsubishi Tanabe Pharma Co., Daiichi Sankyo Inc., Astellas Pharma Inc., Ono Pharmaceutical Co., MSD, Shionogi, Pharma, and Taisho Toyama Pharmaceutical Co. Hidenori Yoshii has nothing to disclose. Masahiko Gosho received lecture and/ or consultant fees from Daiichi Sankyo Company, Ltd., Novartis, and Tiho Pharma K.K., received travel fees from Takeda Pharmaceutical Co., and received manuscript fees from Kowa Co. Ltd. Hitoshi Ishii received lecture and/or consultant fees from Takeda Pharmaceutical Company Ltd., Eli Lilly Japan K.K., Sanofi K.K., Merck \& Co. Inc., Astellas Pharma Inc., Novartis Pharma K.K., Mitsubishi Tanabe Pharma Corporation, Daiichi Sankyo Company Ltd., Ono Pharmaceutical Co. Ltd., AstraZeneca K.K. Taisho Toyama Pharmaceutical Co. Ltd., Shionogi \& Co., Kowa Pharmaceutical Co. Ltd., Boehringer Ingelheim, Novo Nordisk Pharma Ltd., Sumitomo Dainippon Pharma, and Kyowa Hakko Kirin Co. Ltd, unrelated to the submitted work. Iichiro Shimomura received lecture fees from Astellas Pharma Inc., AstraZeneca K.K., MSD K.K., Ono Pharmaceutical Co., Kyowa Hakko Kirin Co., Kowa Pharmaceutical Co., Sanofi K.K., Sanwa Kagaku Kenkyusho Co., Daiichi Sankyo Co., Takeda Pharma K.K., Mitsubishi Tanabe Pharma Co., Teijin Pharma, Eli Lilly Japan K.K., Nippon Boehringer Ingelheim Co., Novartis Pharma K.K., Novo Nordisk Pharma, Bayer Yakuhin, Pfizer Japan Inc., Bristol-Myers K.K., Mochida Pharmaceutical Co., Shionogi \& Co., and Taisho Toyama Pharmaceutical Co., and research funds from Astellas Pharma Inc., AstraZeneca K.K., Eisai Co., MSD K.K, Otsuka Pharmaceutical Co., Ono Pharmaceutical Co., Kaken Pharmaceutical Co., Kissei Pharmaceutical Co., Kyowa Hakko Kirin Co., Sanofi K.K., Shionogi \& Co., Daiichi Sankyo Co., Dainippon Sumitomo Pharma Co., Takeda Pharma K.K., Mitsubishi Tanabe Pharma Co., Teijin Pharma, Nippon Boehringer Ingelheim Co., Novartis Pharma K.K., Novo Nordisk Pharma, Pfizer Japan Inc., Bristol-Myers K.K., Mochida Pharmaceutical Co., Eli Lilly Japan K.K, Kowa Co., Ltd., Kowa Pharmaceutical Co., and Taisho Toyama Pharmaceutical Co. Hirotaka Watada received lecture fees from Novo Nordisk, Inc., Eli Lilly and Company, Sanofi, Dainippon Sumitomo Pharma Co., Fujifilm, Bayer Health Care, Kissei Pharmaceutical Company, Mochida Pharmaceutical Company, MSD, Takeda Pharmaceutical Company, Boehringer Ingelheim Pharmaceuticals Inc., Daiichi-Sankyo, Ono Pharmaceutical Co. Ltd., Novartis Pharmaceuticals Corporation, Mitsubishi Tanabe Pharma Corporation, AstraZeneca LP, Kyowa Hakko Kirin Company Ltd., Sanwa Kagaku Kenkyusyo Company Ltd., Kowa Company Ltd., Astellas Pharma Inc.; advisory fees from Novo Nordisk, Inc., Mochida Pharma Company, AstraZeneca LP, Kowa Company, Astellas Pharma Inc., Sanofi, Boehringer Ingelheim Pharmaceuticals Inc., MSD, Mitsubishi Tanabe Pharma Corporation, Novartis Pharmaceuticals Corporation, Dainippon Sumitomo Pharma Co, Takeda Pharmaceutical Company, Ono Pharmaceutical Co, Pfizer Inc., and Kowa Company; and research funds from Boehringer Ingelheim, Pfizer, Mochida Pharmaceutical Co., Sanofi-Aventis, Novo Nordisk Pharma, Novartis Pharmaceuticals, Sanwakagaku Kenkyusho, Terumo Corp. Eli Lilly, Mitsubishi Tanabe Pharma, Daiichi Sankyo Inc., Takeda Pharmaceutical Co., MSD, Shionogi, Pharma, Dainippon Sumitomo Pharma, Kissei Pharma, and Astrazeneca.

Compliance with Ethics Guidelines. All procedures followed were in accordance with the ethical standards of the responsible committee on human experimentation (institutional and national) and with the Helsinki Declaration of 1964, as revised in 2013. Informed consent was obtained from all patients for being included in the study.

Data Availability. The analyzed datasets are available from the corresponding author on reasonable request.

Open Access. This article is distributed under the terms of the Creative Commons Attribution-NonCommercial 4.0 International License (http://creativecommons.org/licenses/by-nc/4. $0 /$ ), which permits any noncommercial use, 
distribution, and reproduction in any medium, provided you give appropriate credit to the original author(s) and the source, provide a link to the Creative Commons license, and indicate if changes were made.

\section{REFERENCES}

1. Ishii H, Anderson JH Jr, Yamamura A, Takeuchi M, Ikeda I. Improvement of glycemic control and quality-of-life by insulin lispro therapy: assessing benefits by ITR-QOL questionnaires. Diabetes Res Clin Pract. 2008;81:169-78.

2. Ho PM, Rumsfeld JS, Masoudi FA, et al. Effect of medication nonadherence on hospitalization and mortality among patients with diabetes mellitus. Arch Intern Med. 2006;166:1836-41.

3. American Diabetes Association. 8. Pharmacologic approaches to glycemic treatment. Diabetes Care. 2017;40:S64-74.

4. Wright RJ, Frier BM. Vascular disease and diabetes: is hypoglycaemia an aggravating factor? Diabetes Metab Res Rev. 2008;24:353-63.

5. Strowig SM, Aviles-Santa ML, Raskin P. Comparison of insulin monotherapy and combination therapy with insulin and metformin or insulin and troglitazone in type 2 diabetes. Diabetes Care. 2002;25:1691-8.

6. Nemoto M, Tajima N, Kawamori R. Efficacy of combined use of miglitol in type 2 diabetes patients receiving insulin therapy-placebo-controlled double-blind comparative study. Acta Diabetol. 2011;48:15-20.

7. Jacob AN, Salinas K, Adams-Huet B, Raskin P. Weight gain in type 2 diabetes mellitus. Diabetes Obes Metab. 2007;9:386-93.

8. Ose H, Fukui M, Kitagawa Y, et al. Efficacy of glimepiride in patients with poorly controlled insulin-treated type 2 diabetes mellitus. Endocr J. 2005;52:563-9.

9. Ahmed I, Goldstein BJ. Cardiovascular risk in the spectrum of type 2 diabetes mellitus. Mt Sinai J Med N Y. 2006;73:759-68.

10. Katsuno T, Ikeda H, Ida K, Miyagawa J, Namba M. Add-on therapy with the DPP-4 inhibitor sitagliptin improves glycemic control in insulin-treated Japanese patients with type 2 diabetes mellitus. Endocr J. 2013;60:733-42.

11. Shimoda S, Iwashita S, Ichimori S, et al. Efficacy and safety of sitagliptin as add-on therapy on glycemic control and blood glucose fluctuation in Japanese type 2 diabetes subjects ongoing with multiple daily insulin injections therapy. Endocr J. 2013;60:1207-14.

12. Sakamoto Y, Oyama J, Ikeda $H$, et al. Effects of sitagliptin beyond glycemic control: focus on quality of life. Cardiovasc Diabetol. 2013;12:35.

13. Mita T, Katakami N, Shiraiwa T, et al. Sitagliptin attenuates the progression of carotid intima-media thickening in insulin-treated patients with type 2 diabetes: the Sitagliptin Preventive Study of Intima-Media Thickness Evaluation (SPIKE): a randomized controlled trial. Diabetes Care. 2016;39:455.

14. Sato S, Saisho Y, Kou K, et al. Efficacy and safety of sitagliptin added to insulin in Japanese patients with type 2 diabetes: the EDIT randomized trial. PLoS One. 2015;10:e0121988.

15. Mita T, Katakami N, Shiraiwa $\mathrm{T}$, et al. Rationale, design, and baseline characteristics of a clinical trial for prevention of atherosclerosis in patients with insulin-treated type 2 diabetes mellitus using DPP-4 inhibitor: the Sitagliptin Preventive study of Intima-media thickness Evaluation (SPIKE). Diabetol Metab Syndr. 2014;6:35.

16. Japan Diabetes Society. Araki E, Iwamoto Y, Kadowaki T, Kashiwagi A, Kitaoka M, Nanjo K, Tajima $\mathrm{N}$, editors. Treatment guide for diabetes. Tokyo: Bunkodo; 2010.

17. Ishii H. Development and psychometric validation of the Diabetes Therapy-Related QOL (DTR-QOL) questionnaire. J Med Econ. 2012;15:556-63.

18. Nunnally JC. Psychometric theory. 2nd ed. New York: McGraw-Hill; 1978.

19. Anderson RT, Girman CJ, Pawaskar MD, et al. Diabetes medication satisfaction tool: a focus on treatment regimens. Diabetes Care. 2009;32:51-3.

20. Kleefstra N, Ubink-Veltmaat LJ, Houweling ST, Groenier KH, Meyboom-de Jong B, Bilo HJ. Cross-sectional relationship between glycaemic control, hyperglycaemic symptoms and quality of life in type 2 diabetes (ZODIAC-2). Neth J Med. 2005;63:215-21. 